\title{
Functional characterization of the human-specific (type II) form of kallikrein 8, a gene involved in learning and memory
}

\author{
Zhi-xiang $\mathrm{Lu}^{1,2,4}$, Qin Huang ${ }^{3,4}$, Bing $\mathrm{Su}^{1,2}$ \\ ${ }^{1}$ State Key Laboratory of Genetic Resources and Evolution, Kunming Institute of Zoology, Chinese Academy of Sciences, Kunming \\ 650223, China; ${ }^{2}$ Kunming Primate Research Center, Chinese Academy of Sciences, Kunming 650223, China; ${ }^{3}$ Institute of Bio- \\ chemistry and Cell Biology, Shanghai Institutes for Biological Sciences, Chinese Academy of Sciences, Shanghai 200031, China; \\ ${ }^{4}$ Graduate School of Chinese Academy of Sciences, Beijing 100049, China
}

Kallikrein 8 (KLK8) is a serine protease functioning in the central nervous system, and essential in many aspects of neuronal activities. Sequence comparison and gene expression analysis among diverse primate species identified a human-specific splice form of $K L K 8$ (type II) with preferential expression in the human brain, which may contribute to the origin of human cognition. To gain insights into the physiological and biochemical role of this novel form, we conducted functional analyses of human type II KLK8. Our results show that type II $K L K 8$ is abundantly expressed in human embryonic stem cells and in embryo brain samples, suggesting a potential role in embryogenesis. There are dramatic expression variations in different individuals and brain regions, which is a reflection of its dynamic role in neural activities. Furthermore, the transcription start site (TSS) of $K L K \delta$ is tissue-specific, with a brain-specific TSS found in humans indicating functional specialization. Our in vitro biochemical assay shows that there is a type IIspecific intermediate protein form, although the processed end-point enzymes are the same for both type I and type II KLK8, suggesting that the emergence of type II KLK8 in the human brain likely leads to functional modifications of KLK8.

Keywords: type II KLK8, alternative splicing, cognition, human evolution

Cell Research (2009) 19:259-267. doi: 10.1038/cr.2009.4; published online 6 January 2009

\section{Introduction}

Alternative splicing (AS) is critical for the expansion of proteomic diversity and the regulation of gene expression [1]. It is also one of the major mechanisms in the genome for the creation of new proteins during evolution $[2,3]$. The human brain, widely accepted as a product of adaptive evolution, preferably utilizes this strategy in its functional complexity [4]. Experimental studies of individual genes have revealed many cases of functional differences between different splice forms during ontogenesis. Sometimes, the ratio change of the expressed splice variants may have pathogenic consequences [5, $6]$. The enlarged brain and highly improved cognitive

Correspondence: Bing $\mathrm{Su}$

Tel: +86-871-5120212

E-mail:sub@mail.kiz.ac.cn

Received 27 June 2008; revised 30 June 2008; accepted 30 June 2008; published online 6 January 2009 abilities are among the most significant changes during the origin of our own species. Hence, elucidating the functional consequences of human-specific splice forms in the central nervous system (CNS) is a prerequisite for improved understanding of the functional evolution of human cognition.

KLK 8 (also called neuropsin, MIM\# 605644) is a secreted-type serine protease preferentially expressed in CNS, and was initially cloned from the mouse hippocampus [7]. Electrophysiology and behavioral studies have implicated its involvement in hippocampal plasticity and kindling epileptogenesis [8-10].

Human KLK8 was originally cloned as the human ortholog of the mouse brain protease [11] and is known to be associated with diseases such as ovarian cancer and Alzheimer's disease [12-14]. It is also a favorable prognostic marker in clinical diagnosis $[15,16]$. This gene is alternatively spliced in the human brain. Mitsui et al. [17] reported two types of human KLK8 cDNAs in the brain. One of them (type I) is homologous with the 
mouse counterpart while the other (type II) is absent in the mouse (Figure 1). The two transcripts differ only in their exon 3 sequences. Type II includes extra 45 amino acids at the $\mathrm{N}$-terminus of exon 3 due to alternative splicing. In vitro splicing assay has shown that a human-specific point mutation led to the origin of this novel form in the human brain. Moreover, the chimpanzee sequence was able to express type II KLK8 when the human-like mutation was introduced [18]. Similar to type I, humanspecific type II KLK8 is also abundantly expressed in the CNS of both fetuses and adults, as well as in the placenta $[17,18]$. However, little is known about the biological function of type II KLK8, even though earlier studies have proposed biochemical and enzymatic properties for human type I KLK8 $[19,20]$.

With the use of RT-PCR and parallel biochemical assays, we show that the expression of human type II KLK8 starts during embryogenesis and that there are large inter-individual and inter-brain region variations.
The in vitro biochemical assay reveals that type I and type II forms are released in a cell type-dependent manner. Additionally, type II is more efficiently secreted in High Five cells, though the final active proteins are the same for both type I and type II KLK8, suggesting functional modifications for type II in the human brain.

\section{Results}

Temporal-spatial expression of type II KLK8 in human brain

Our earlier studies using in vitro splicing assays have demonstrated that generation of type II KLK8, which is caused by a single nucleotide substitution, was fixed during human evolution and is therefore absent in nonhuman primates [18]. To further understand the detailed expression pattern of type II we conducted a study, in which different developmental stages and different brain regions in humans were examined. A total of three em-

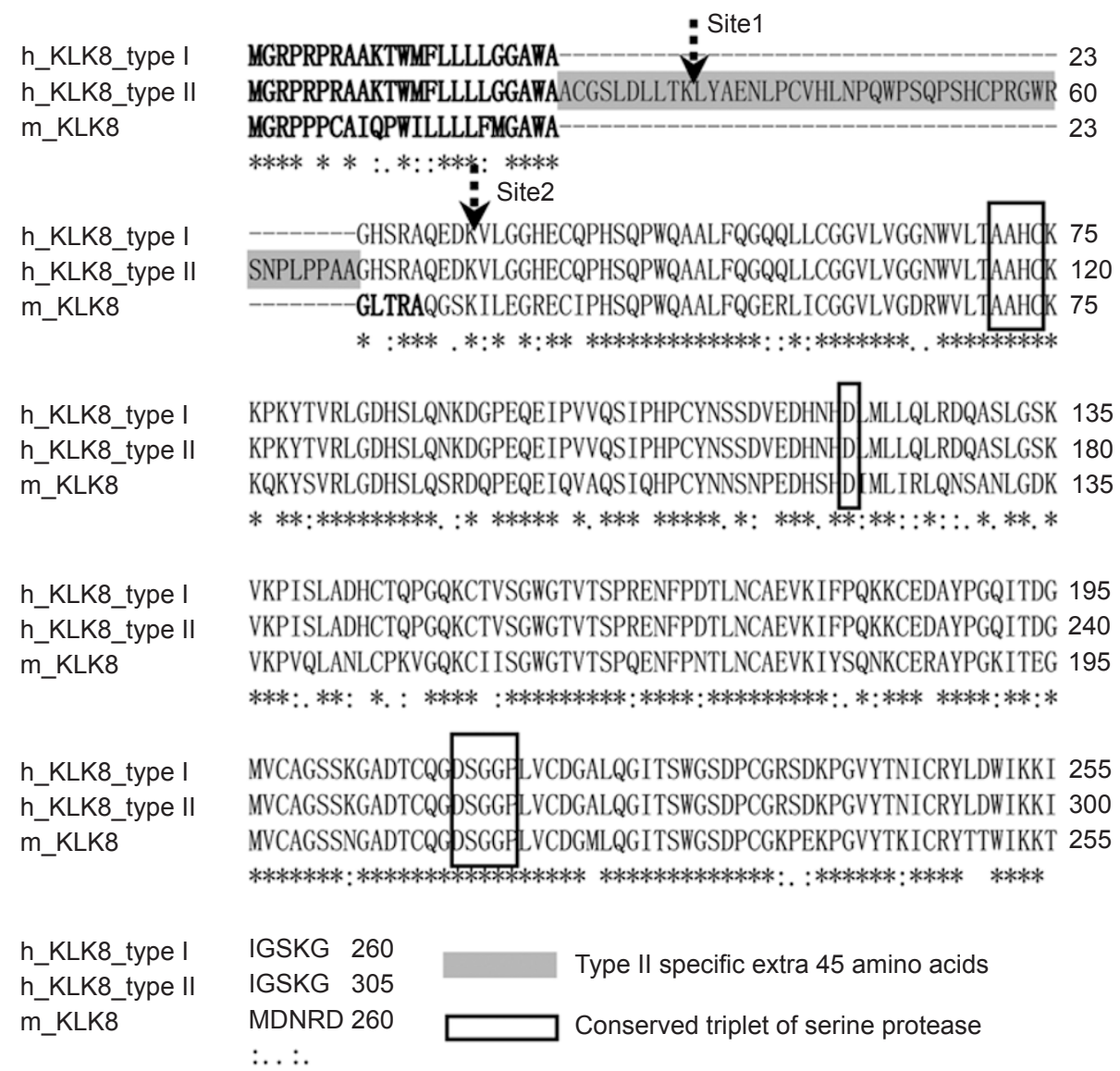

Figure 1 Alignment of protein sequences of human and mouse KLK8. Site 1 and Site 2 refer to the endoprotease sites. KLK8 activation occurs after endoprotease cleaves next to the lysine residue at the site 2 . Bold letters indicate species-specific secretion signal peptides of KLK8. The human sequence exhibits a 23 amino acid signal peptide, while the mouse sequence possesses a 28 amino acid signal peptide [19, 32]. 
bryos and nine adult brain tissue samples were tested and a complex expression pattern was observed for type II (Figure 2A and 2B). For example, of the three embryo brain samples tested, two expressed type II (HE02 and HE03) and the other lacked its expression (HE01). In the nine adult brain samples examined, only four showed type II expression. In addition to the inter-individual variation, type II is also characterized by inter-regional variations in brain expression. For example, in embryo brain sample, HE03 expression was detected in the midbrain and posterior brain, but absent from the frontal brain. Similar to its ancestral type (type I) [8, 21-23], type II was also expressed in a temporal-spatial manner. We also tested human embryonic stem cells and observed an abundance of type II expression, suggesting a potential role in early embryogenesis (Figure 2C).

\section{Type II KLK8 transcript can produce an intact protein in} vitro

Structural modeling of splice variants from genes of $1 \%$ of the genome surveyed by the ENCODE consortium has predicted that many alternative splicing events are unlikely to result in correct protein folding [24], which may lead to instability, degradation and malfunction [25]. To prove that the novel form of human $K L K 8$ could be normally translated, we cloned the full length coding sequence of $K L K 8$. Western blot analysis detected two bands corresponding to type I and type II KLK8, respectively (Figure 2D), indicating that type II KLK8 is likely to be produced as an intact protein.
Brain-specific TSS in KLK8 expression

Recent reports have shown that gene expression can be regulated by alternative UTRs [26, 27], which could also affect alternative splicing through differential promoter usage [28-30]. Our earlier data have suggested the divergence of $K L K 8$ promoter sequences and that KLK8 TSS differs between human and non-human primate brains [18]. With the use of $5^{\prime}$ RACE, we determined the TSS for both type I and type II $K L K 8$ in different tissue samples from human and rhesus macaque (Macaca mulatta) (Figure 3). Although type I and type II share the same TSS in humans and lack a splice-form-specific promoter, the brain TSSs in humans and rhesus macaques differ [18] and exhibit tissue specificity. This observation suggests that during the origin of type II, there was a brain-specific expression regulation change, although type I KLK8 is commonly expressed in many tissues under normal and pathological conditions [31].

Similar secretion and activation efficiency between type I and type II KLK8

Earlier studies have demonstrated that in vivo activation of KLK8 includes two key steps. First, the Nterminus signal peptide (23 amino acids in humans [20]; 28 amino acids in mice [32]) (Figure 1) is removed after KLK8 is translocated into the endoplasmic reticulum in the host cell (from pre-pro-protein to pro-protein). Second, the pro-protein is enzymatically processed by endoprotease (site 1 and 2 in Figure 1) and becomes the active form. Human KLK 8 type II includes extra 45

A

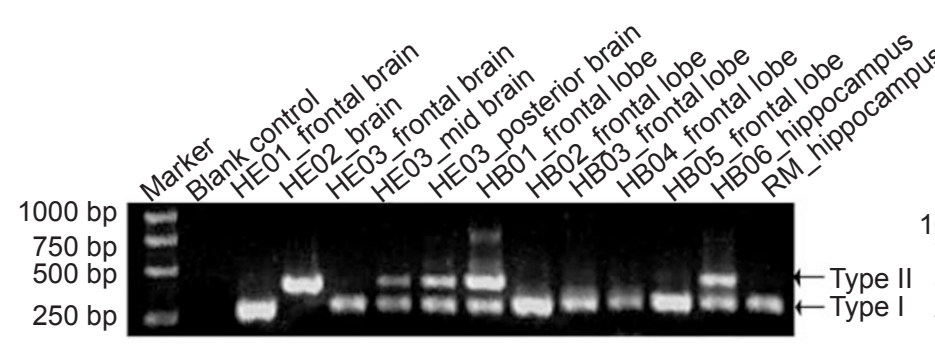

C

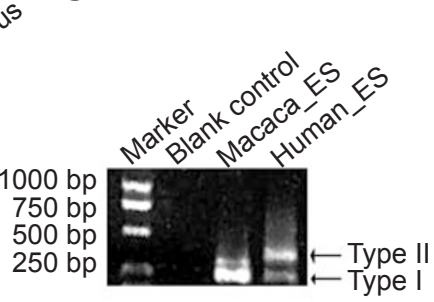

B

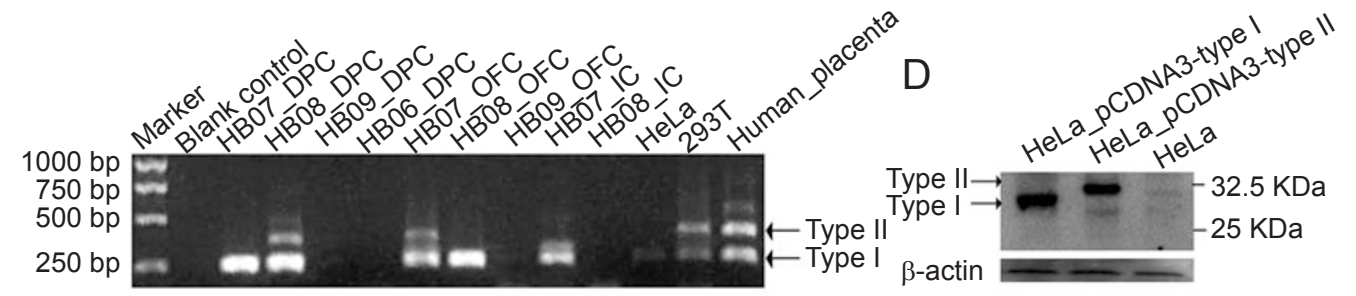

Figure 2 The expression pattern of human KLK8, and western blot of transfected HeLa cells. (A-C) the electromorph of the RT-PCR results. The house-keeping gene (actin) was used to confirm the integrity of our CDNA (data not shown). HE, human fetal brain; HB, human adult brain; DPC, dorsal prefrontal cortex; OFC, orbital frontal cortex; IC, inferior convexity; ES, embryo stem cell. The numbers indicate different individuals. (D) Western blot of the KLK8 isoforms, i.e. type I and type II. 
amino acids compared with the ancestral type I; this 45 amino acid insertion creates a novel endoprotease recognition site (site 1 in Figure 1) and it does not disrupt the signal peptide region and the triplet (His-Asp-Ser) serine protease domain $[20,32]$ (Figure 1).

To compare the activation process and enzyme activity of type I and type II KLK8, we produced the recombinant full-length type I and type II proteins in parallel using the baculovirus insect cell system. As KLK8 is a secreted protein, we first tested the secretion efficiency of the two types. Using the intracellular KLK8 protein as the loading control, we detected a significant secretion difference, with type II possessing a much higher secretion efficiency of pro-proteins than type I in High Five cells (Figure 4). However, when using HeLa and 293 cell lines, we found that the secretion of these two forms was cell-type dependent. In HeLa cells, we did not detect secreted proteins in the culture medium. In 293 cells, there was no significant difference of secretion efficiency between type I and type II (Figure 4).

The enzyme activity assay using synthetic substrates shows that the type II pro-protein has very low amidolyt- ic activity (Figure 5A-5C), as does the type I pro-protein (data not shown). The medium from infected insect cells has high amidolytic activity following endoprotease processing (protease 1 (EC 3.4.21.30) from Achromobacter lyticus is used) for both type I [32] and type II (Figure $5 \mathrm{~A}-5 \mathrm{C}$, type I was not shown). The incubation duration analysis with protease 1 indicates that the final active forms are the same for type I and type II KLK8. In other words, the extra 45 amino acids of type II would be removed during the enzyme activation process, resulting in the same end-point enzyme as that of type I (Figure 5D).

Although the final active enzyme of type II KLK8 is not a novel form, there is one endoprotease site in the extra 45 amino acid region, and KLK8 activation occurs only after the endoprotease cleaves next to the Lys residue at the Site 2. Therefore, an intermediate protein form exists for type II (but not for type I) during the activation process, which was detected in the site-mutation assay (Figure 5E). As there are only nine amino acids upstream of the site 1 cleavage site (Figure 1), we could not detect the difference in molecular weights in our western blot analysis (Figure 5D and 5E). To identify the biochemical

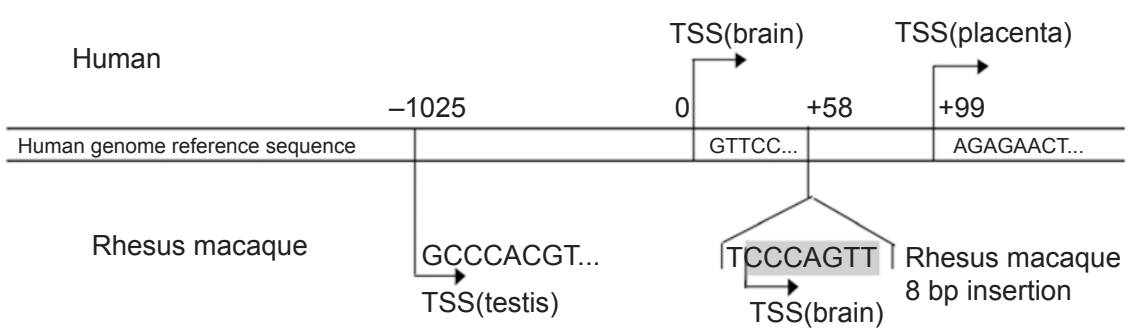

Figure 3 Changes of $K L K 8$ transcription start site (TSS) in different tissues and different species. The brain TSSs of human and rhesus macaque from earlier studies $[17,18]$.
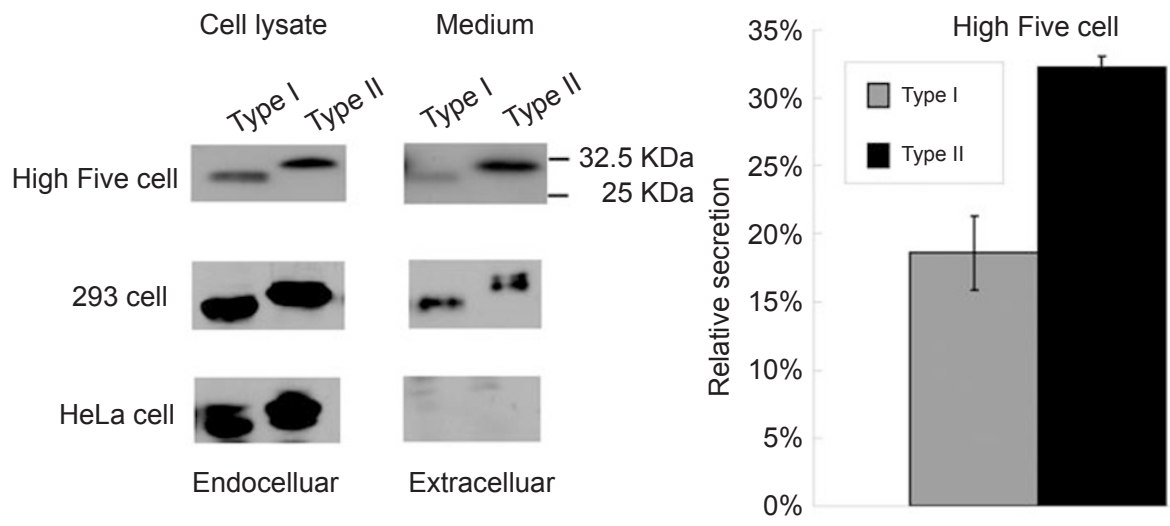

Figure 4 Relative efficiency of secretion between the two KLK8 isoforms in different cell types. The amount of the proteins was quantified based on the band intensities of western blot (see methods, the relative secretion is given as means \pm SD. Type I, $18.6 \% \pm 2.7 \%$; type II, $32.3 \% \pm 0.8 \%$.). 
A

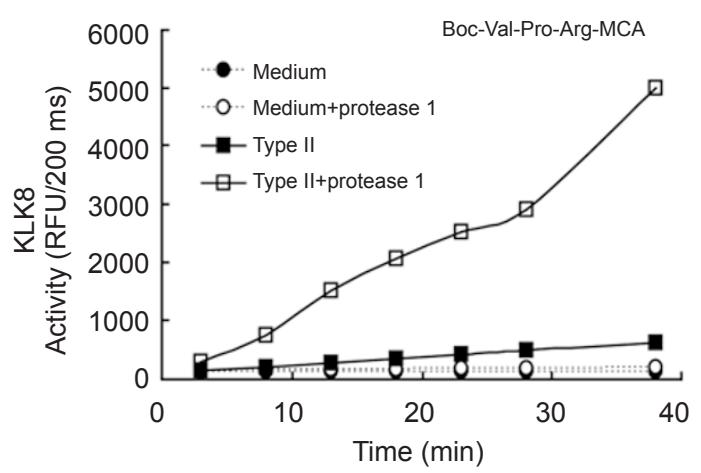

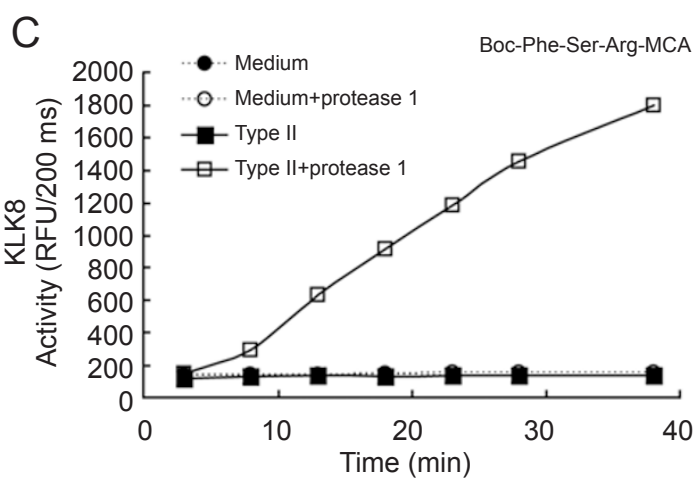

$\mathrm{D}$

B
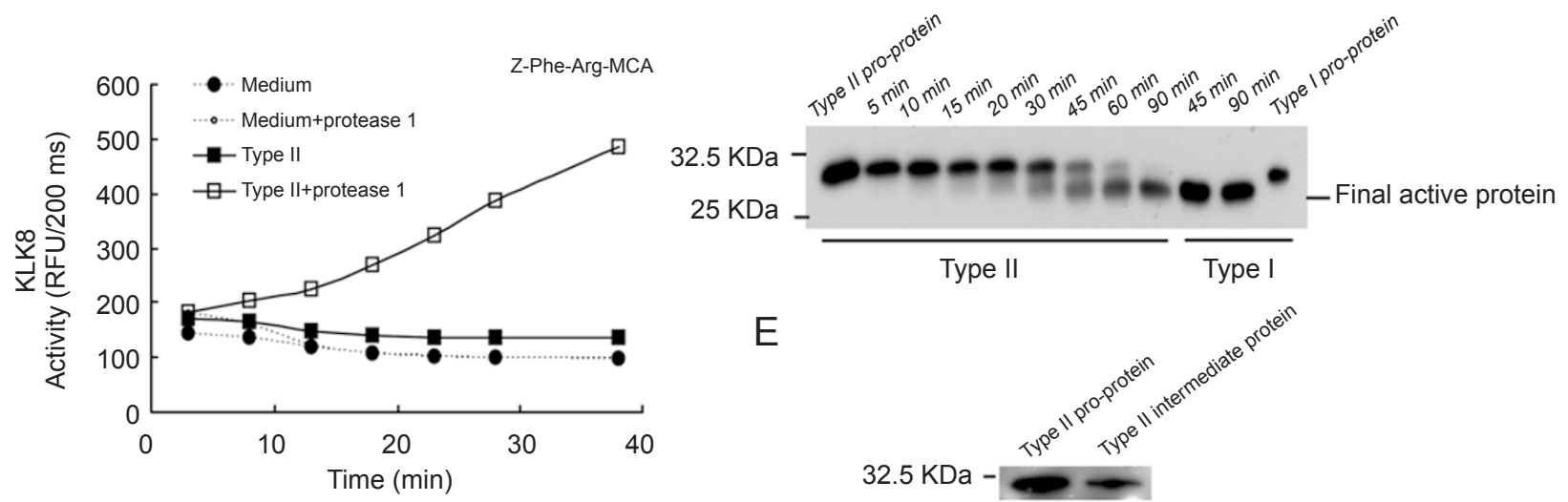

E

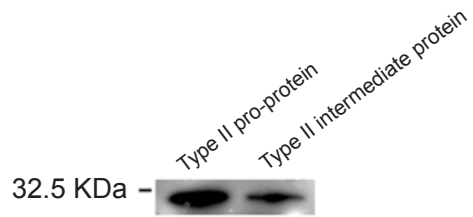

Figure $\mathbf{5}$ The test of activation process and enzyme activity of type II KLK8. (A-C) Trypsin-like activity of pro-protein type II and protease 1 treated pro-protein type II. Three different synthetic substrates were used. (D) Process of pro-protein type II KLK8 activation. (E) Western blot of the intermediate type II pro-protein. The intermediate type II pro-protein was produced by type II_mu2 mutant protein, which was incubated with protease 1 for sufficient time. RFU, relative fluorescence units.

feature of the intermediate type II form, we mutated the original endoprotease site (from Lys 77 to His 77 at site 2 in Figure 1) so that the resulting type II_mu2 only gave rise to the intermediate type II form when it was processed by protease 1 . The enzyme assay reveals that this type II intermediate form has very low amidolytic activity, like the other pro-protein forms (Figure 6A). We next checked the activation efficiency of the three different pro-protein forms, including the type I pro-protein, type II pro-protein and type II_Mu1 pro-protein (point mutation at site 1 in Figure 1, from Lys33 to His33; this mutation was designed to eliminate the novel endoprotease site). Our data indicate that all three pro-protein forms reach their highest activity at 90 minutes after incubation with protease 1 (Figure 6B), and that their activation efficiencies are the same.

\section{Discussion}

KLK8 is a trypsin-like serine protease abundantly expressed in the CNS, especially in the hippocampus and associated limbic structures. It has been reported earlier that the shuttle-box avoidance learning was retarded at 7 days after oxidative stress, which reduced KLK8 mRNA to $60 \%$ of the control level in mouse [9]. KLK8 is also essential for the early processes of memory acquisition and Schaffer collateral long-term potentiation in the adult mouse hippocampus in vivo [10]. This functionally critical brain protease has evolved a novel form in humans. Combining data from Mitsui's [17] northern blot for detecting this novel transcript in tissues and Sher's [16] siRNA assay for analyzing endogenous human KLK8 type II protein in cell lines, our functional characterization suggests that this novel form is likely to be functional in the human brain.

Earlier studies demonstrated that mouse KLK8 ortholog was expressed in an activity-dependent manner and could be induced by incision through intraperitoneal kainite injection or kindling $[21,22,33]$. As expected, the KLK8 type II transcript is also regulated in a temporal-spatial manner, and there is a brain specific TSS in the human brain. The expression of type II KLK8 in human 
A

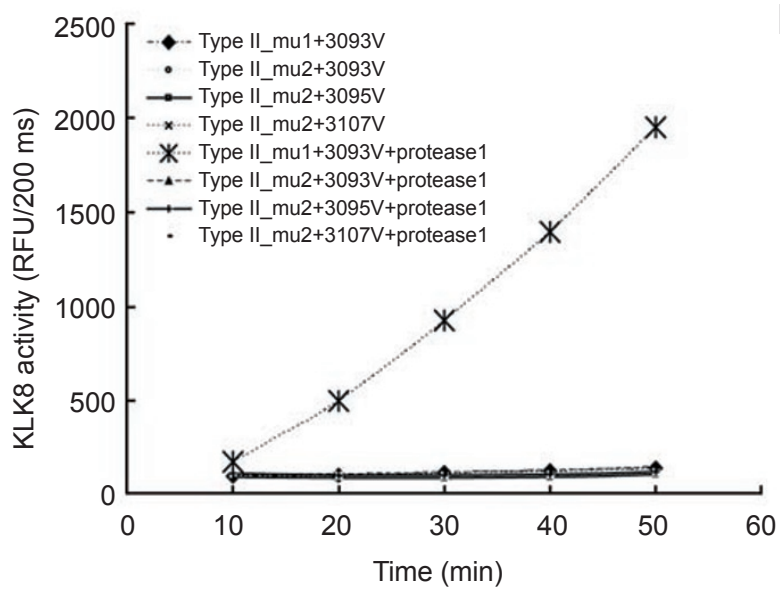

B

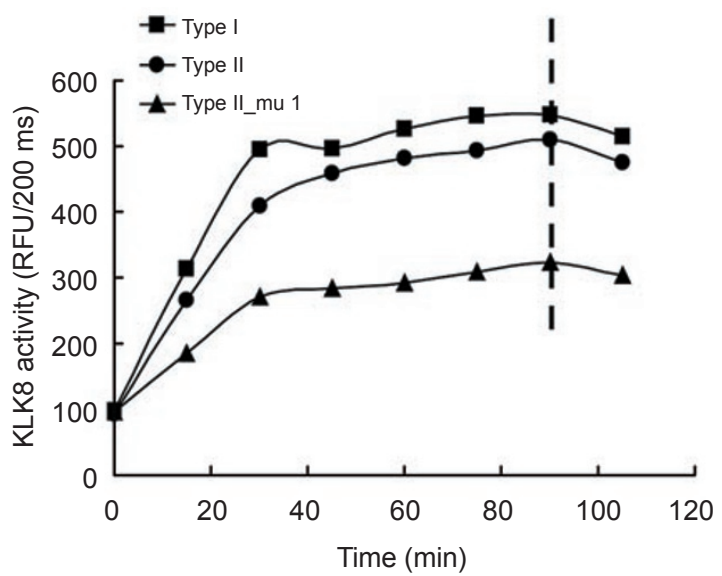

Figure 6 Enzyme activity of the intermediate type II pro-protein and activation efficiency of KLK8 isoforms. (A) Trypsin-like activity of the intermediate type II (produced by type II_mu2 mutant, point mutation at site 2 in Figure 1, from Lys77 to His77). 3093V, 3107V and 3095V are different synthetic substrates of KLK8 (see Materials and Methods). The type II_mu1 mutant (point mutation at site 1 in Figure 1, from Lys33 to His33) was used as the positive control during protease 1 treatment. (B) The effect of the extra 45 amino acids of type II KLK8 on activation process. The same molar pro-proteins were tested here. Incubation time for the protein reaching its highest activity is assumed, as pro-protein has entirely transformed into its final active form. Reaction samples were also subjected to western blot to confirm cleavage process (data not shown). Different RFUs of the three proteins' highest activities are due to different activity units per mg proteins we produced in vitro.

embryonic stem cells suggests that it might contribute to the remodeling of extracellular components after decidualization during embryonic development [34, 35]. The expression of type II in the embryo brain also suggests that the novel splice form is likely to be a multifunctional protein engaging in varied processes during development, such as neurite outgrowth [36, 37]. Additionally, the expression of type II seems to be precisely regulated by both cis-elements and trans-factors. Our earlier data have revealed that activity of the $K L K 8$ gene promoter between humans and chimpanzees was different in 293T cells, though no difference was observed in HeLa cells. In this study, we show that type II KLK8 is expressed in 293 T cells, but not in HeLa cells (Figure 2B). Further studies are needed to dissect the regulatory mechanism of type II KLK8 expression.

During the secretion process, the human KLK8 zymogen is formed by removing the 23 amino acid signal peptide at the N-terminus [20], while the mouse zymogen is created using a different site [32] (Figure 1). It seems that during human evolution, natural selection acted to preserve the extra 45 amino acids of the type II pro-protein form, while conserving the original serine protease domain. In addition, the Kyte-Doolittle hydropathy plot and secondary structure analysis show that the extra 45 amino acid domain is hydrophilic and forms a small $\alpha$ helix. Our enzyme activity assay also demonstrates that this novel type II form can produce active protease, indi- cating the maintenance of the proper structure of type II pro-protein.

We also tested the constitutive secretion of KLK8 in transfected cells. Secretion of these two forms varied in different cells (Figure 4). Oka et al. [38] have shown that the release of transfected KLK8 is significantly stimulated by a high $\mathrm{K}^{+}$medium and that this stimulated secretion is dependent on the presence of calcium, suggesting that KLK 8 could be secreted in a regulated manner. We could not rule out the possibility that type II KLK8 was more efficiently secreted only in High Five cells. To elucidate the function of this newly evolved type II KLK8 in human brain in vivo, further studies should focus on identifying which type of neuron in the human brain expresses type II KLK8 and how its expression responds to stimuli.

Additionally, post-translational regulation of KLK8 is critical for its normal function. For example, during the early phase of LTP, KLK8 needs to be extracellularly activated to modify the synaptic adhesion [39]. Our assays using recombinant type II and mutant proteins suggest that type II has an intermediate protein form; although without protease activity, it might regulate the active protein through protein-protein interactions in the zymogen pool. Further experimental studies are required to determine the precise regulation mechanism of type II KLK8 and to reveal the role of type II intermediate protein in the zymogen pool. 


\section{Materials and Methods}

\section{Samples and RT-PCR}

For gene expression pattern analysis we collected aborted embryos (HE01: 20 weeks; HE02: 16 weeks; HE03: 23 weeks) and postmortem human brain tissues (28-50 years old adults) from local hospitals. The human protocol was approved by these institutions. Informed consent was obtained from all human subjects.

The total RNAs were extracted using TRIzol (Invitrogen, Carlsbad, CA, USA). The RNA extraction tests indicated that none of the samples showed visible RNA degradation. For cDNA synthesis, $1 \mu \mathrm{g}$ of DNase I-treated (Fermentas, Hanover, MD, USA) total RNA was reverse transcribed using Oligo-d $(\mathrm{T})_{22}$ primer and ominiscript reverse transcriptase enzyme (Qiagen, Valencia, CA, USA). The primers designed to detect $K L K 8$-splicing isoform were in accordance with our methods published earlier [18].

\section{Human KLK8 cDNA cloning and western blot analysis}

For cloning cDNAs of human type I and type II $K L K 8$, human placenta total RNA was extracted using TRIzol (Invitrogen), One step RT-PCR was carried out at $50{ }^{\circ} \mathrm{C}$ for $30 \mathrm{~min}$, at $95^{\circ} \mathrm{C}$ for 15 min, at $94{ }^{\circ} \mathrm{C}$ for $30 \mathrm{~s}, 61{ }^{\circ} \mathrm{C}$ for $30 \mathrm{~s}$ and $72{ }^{\circ} \mathrm{C}$ for $40 \mathrm{~s}$, for 32 cycles using appropriately diluted RNA as a template (Qiagen OneStep RT-PCR Kit). One step RT-PCR products were used to carry out another nested PCR. Final products were cloned into pMD19 simple-T-vector (Takara, Tokyo, Japan) then digested by BamHI and HindIII and subcloned into pcDNA-3 vector (Invitrogen). Final constructs were confirmed by sequencing (ABI-3130 automatic sequencer, Boston, MA, USA).

Approximately $40 \%$ confluent HeLa cells were grown overnight in DMEM media (Gibco, Rockville, MD, USA) containing $10 \%$ fetal bovine serum (Hyclone, Logan, UT, USA) in 6-well plates, and then they were transfected with $2 \mu \mathrm{g}$ of plasmid DNA using Lipofectamine 2 000, following the manufacturer's protocol (Invitrogen). Forty-eight hours after transfection, HeLa cells were washed using PBS (phosphate buffer) twice and then lysed. Western blot was performed according to the conventional method (Total protein was subjected to $10 \%$ SDS-PAGE analysis under reducing conditions). The cloning primer sequences and the western blot antibodies are described below:

NP 131 F 5'-GGT CCG AAT CAG TAG GTG AC-3' NP_1122_R 5'-ACC AGA GAG TTG TGA GTT TAT TAA G-3'

KLK8 F 5'-CGG GAT CCT CAC CAT GGG ACG CCC-3'

KLK8 ${ }^{-}$R 5'-GGT ACC AAG CTT GCC CTT GCT GCC TAT GAT C-3'

We used KLK8 specific antibodies for western blot analysis at adequate dilutions as follows: anti-human KLK8 mouse monoclonal antibody 1:3000 (Neuromics, Edina, MN, USA, catalog number: MO15032), anti- $\beta$-actin mouse monoclonal antibody 1:3 000 (Abcam, Cambridge, MA, USA, clone number: mAbcam 8226), anti-mouse IgG (h\&l) rabbit polyclonal antibody HRP-conjugated 1:5 000 (Bethyl, Montgomery, TX, USA, catalog number: A90$117 \mathrm{P})$.

\section{5'RACE of KLK8 gene}

5'RACE was carried out using a SMART ${ }^{\mathrm{TM}}$ RACE cDNA Amplification Kit (Clontech, Polo Alto, CA, USA). Primers and PCR conditions used were according our methods published earlier [18].
Fragments were cloned into pMD19simple-T-vector (Takara) and sequenced. GenBank accession numbers: EU350537, EU350538 and EU350539.

Recombinant KLK8 preparation and enzyme activity assay

For enzyme activity assay, the synthetic substrates (3104V: Boc-Val-Leu-Lys-MCA, 3093V: Boc-Val-Pro-Arg-MCA, 3095V: Z-Phe-Arg-MCA, 3107V: Boc-Phe-Ser-Arg-MCA) were obtained from Peptide Institute Inc. (Tokyo, Japan). The KLK8 endoprotease protease 1 (Achromobacter lyticus, EC 3.4.21.30) was obtained from Wako Pure Chemical Inc. (Osaka, Japan). TLCK (1-chloro-3-tosylamido-7-amina-2-heptanone $\mathrm{HCl}$ ), obtained from AppliChem Inc. (Darmstadt, Germany), was protease 1 specific inhibitor but did not show effects on KLK8 activity [20].

Two wild-type isoforms of KLK8 were subcloned into pFastBAC1 (primers: KLK8_bac_F 5'-CGG GAT CCT CAC CAT GGG ACG CCC-3', KLK 8 _bac_R 5'-ACG CTC GAG TCA ATG ATG ATG ATG ATG ATG GCC CTT GCT GCC-3'), the other two mutation-associated constructs were undertaken by primermediated site-directed mutagenesis. The final constructs were confirmed by sequencing. All constructs were integrated in bacmid DNA according to the instruction manual (Bac-to-Bac Baculovirus Expression System, Invitrogen). Bacmid DNA was isolated using the S.N.A.P ${ }^{\mathrm{TM}}$ MidiPrep Kit (Invitrogen). Then bacmid DNA was transfected into Sf9 cell in SF900 II -SFM medium (Gibco), 2-8 $\times$ $10^{7} \mathrm{pfu} / \mathrm{ml}$ titer baculovirus were produced finally by amplification. High Five cells at a density of $1 \times 10^{6}$ cells $/ \mathrm{ml}$ were infected with recombinant baculovirus at a multiplicity of infection of about two. Three to five days post-infection, the incubation Express Five $^{\circledR}$ SFM medium (Gibco) was adjusted to $\mathrm{pH} 8.2$ and the cells were harvested by removing the cell debris by centrifugation. The pro-protein containing SFM medium was collected for further enzyme activity assay.

To detect the secretion difference, corresponding transfected High Five cells were harvested and lysed. Using endocellular KLK8 proteins as loading controls, the proportional volumes of extracellular KLK8 medium were used for western blot. High Five cells were infected twice independently. The band intensities of western blot were quantified by the Glyko BandScan 4.30 software package (Novato, CA, USA). The 293 and HeLa cell lines secretion experiments were conducted using the same protocol as described above (see Materials and methods: Human KLK 8 cDNA cloning and western blot analysis) except that the cell medium was also collected for detecting secretion efficiency.

To detect enzyme activity, the Express Five ${ }^{\circledR}$ SFM medium (pH 8.2) containing 40-150 ng KLK8 pro-protein was pre-warmed at $37{ }^{\circ} \mathrm{C}$ for $30 \mathrm{~min}$ in a black 96-well micro titer plate, then $1 \mu \mathrm{l}$ of $10 \mathrm{mM}$ substrate solution (dissolved in DMSO) was added into each well and gently mixed. The fluorescence of released MCA was monitored at different times (excitation at $355 \mathrm{~nm}$, emission at $460 \mathrm{~nm}$ ) using Fluoroskan Ascent FL 2.5 instrument (Thermo Electron Corp., Waltham, MA, USA). The cleaved protein activity was measured using the same procedure as above except that the medium containing the recombinant pro-protein was treated by $1 \mu \mathrm{l}$ protease 1 , in each well ( 2 activity unit $/ \mathrm{ml}, \mathrm{pH} 8.3,0.5 \mathrm{M}$ $\mathrm{NaCl}$ and $0.1 \mathrm{M} \mathrm{NaHCO}_{3}$ ), for $10 \mathrm{~min}$ at $37{ }^{\circ} \mathrm{C}$ before synthetic substrates were added. As protease 1 has no enzyme activity on KLK8's synthetic substrates (data not shown), we did not inhibit protease 1 in the medium before we added the synthetic substrates in this enzyme activity experiment. 
To detect the cleavage difference of pro-protein, identical molar pro-proteins (quantified by western blot) were balanced at $37{ }^{\circ} \mathrm{C}$ for $30 \mathrm{~min}$. Then we added $0.45 \mu \mathrm{l}$ ( 2 activity unit $/ \mathrm{ml})$ protease 1 to each reaction mixture in a black 96 -well plate and incubated it at $37^{\circ} \mathrm{C}$ in time-gradient manner. After incubation for the required time duration, we added TCLK inhibitor to each reaction mixture (at a final TCLK concentration of $0.15 \mathrm{mg} / \mathrm{ml}$, this concentration was enough to inhibit all residual protease 1 activity (data not shown)). Finally we added $1.5 \mu \mathrm{KLK} 8$ substrates $(10 \mathrm{mM})$ to each of the reaction mixtures. After another $30 \mathrm{~min}$ incubation at $37^{\circ} \mathrm{C}$, the fluorescence of released MCA was monitored as above. All above enzyme assays were conducted at least twice independently.

\section{Acknowledgments}

We thank Dr Jin-qiu Zhou (Institute of Biochemistry and Cell Biology, SIBS, CAS, China), who kindly provided the High Five cells. We also thank Dr Wei-zhi Ji (Kunming Institute of Zoology, CAS, China), who kindly provided the stem cell cDNA samples. We thank Dr Darren Curnoe (University of New South Wales, Australia) for the critical reading of the manuscript. We are grateful to the technical help of Jia Peng and Hui Zhang (Kunming Institute of Zoology, CAS, China). This study was supported by grants from National Basic Research Program of China (973 Program) (2006CB701506, 2007CB815705), the Chinese Academy of Sciences (KSCX1-YW-R-34), the National Natural Science Foundation of China (30370755, 30525028 and 30630013), and the Natural Science Foundation of Yunnan Province of China.

\section{References}

1 Black DL. Mechanisms of alternative pre-messenger RNA splicing. Annu Rev Biochem 2003; 72:291-336.

2 Blencowe BJ. Alternative splicing: new insights from global analyses. Cell 2006; 126:37-47.

3 Xing Y, Lee C. Evidence of functional selection pressure for alternative splicing events that accelerate evolution of protein subsequences. Proc Natl Acad Sci USA 2005; 102:1352613531.

4 Grabowski PJ. Splicing regulation in neurons: tinkering with cell-specific control. Cell 1998; 92:709-712.

5 Andreadis A. Tau gene alternative splicing: expression patterns, regulation and modulation of function in normal brain and neurodegenerative diseases. Biochim Biophys Acta 2005; 1739:91-103.

6 Lopez AJ. Alternative splicing of pre-mRNA: developmental consequences and mechanisms of regulation. Annu Rev Genet 1998; 32:279-305.

7 Chen ZL, Yoshida S, Kato K, et al. Expression and activitydependent changes of a novel limbic-serine protease gene in the hippocampus. J Neurosci 1995; 15:5088-5097.

8 Momota Y, Yoshida S, Ito J, et al. Blockade of neuropsin, a serine protease, ameliorates kindling epilepsy. Eur J Neurosci 1998; 10:760-764.

9 Akita H, Matsuyama T, Iso H, Sugita M, Yoshida S. Effects of oxidative stress on the expression of limbic-specific protease neuropsin and avoidance learning in mice. Brain Res 1997; 769:86-96.

10 Tamura H, Ishikawa Y, Hino $\mathrm{N}$, et al. Neuropsin is essential for early processes of memory acquisition and Schaffer collateral long-term potentiation in adult mouse hippocampus in vivo. J Physiol 2006; 570:541-551.

11 Yoshida S, Taniguchi M, Hirata A, Shiosaka S. Sequence analysis and expression of human neuropsin cDNA and gene. Gene 1998; 213:9-16.

12 Magklara A, Scorilas A, Katsaros D, et al. The human KLK8 (neuropsin/ovasin) gene: identification of two novel splice variants and its prognostic value in ovarian cancer. Clin Cancer Res 2001; 7:806-811.

13 Borgono CA, Diamandis EP. The emerging roles of human tissue kallikreins in cancer. Nat Rev Cancer 2004; 4:876-890.

14 Shimizu-Okabe C, Yousef GM, Diamandis EP, Yoshida S, Shiosaka S, Fahnestock M. Expression of the kallikrein gene family in normal and Alzheimer's disease brain. Neuroreport 2001; 12:2747-2751.

15 Yousef GM, Obiezu CV, Luo LY, et al. Human tissue kallikreins: from gene structure to function and clinical applications. Adv Clin Chem 2005; 39:11-79.

16 Sher YP, Chou CC, Chou RH, et al. Human kallikrein 8 protease confers a favorable clinical outcome in non-small cell lung cancer by suppressing tumor cell invasiveness. Cancer Res 2006; 66:11763-11770.

17 Mitsui S, Tsuruoka N, Yamashiro K, Nakazato H, Yamaguchi N. A novel form of human neuropsin, a brain-related serine protease, is generated by alternative splicing and is expressed preferentially in human adult brain. Eur J Biochem 1999; 260:627-634.

$18 \mathrm{Lu}$ ZX, Peng J, Su B. A human-specific mutation leads to the origin of a novel splice form of neuropsin (KLK8), a gene involved in learning and memory. Hum Mutat 2007; 28:978-984.

19 Rajapakse S, Ogiwara K, Takano N, Moriyama A, Takahashi T. Biochemical characterization of human kallikrein 8 and its possible involvement in the degradation of extracellular matrix proteins. FEBS Lett 2005; 579:6879-6884.

20 Kishi T, Cloutier SM, Kundig C, Deperthes D, Diamandis EP. Activation and enzymatic characterization of recombinant human kallikrein 8. Biol Chem 2006; 387:723-731.

21 Okabe A, Momota Y, Yoshida S, et al. Kindling induces neuropsin mRNA in the mouse brain. Brain Res 1996; 728:116120.

22 He XP, Shiosaka S, Yoshida S. Expression of neuropsin in oligodendrocytes after injury to the CNS. Neurosci Res 2001; 39:455-462.

23 Suzuki J, Yoshida S, Chen ZL, et al. Ontogeny of neuropsin mRNA expression in the mouse brain. Neurosci Res 1995; 23:345-351.

24 Tress ML, Martelli PL, Frankish A, et al. The implications of alternative splicing in the ENCODE protein complement. Proc Natl Acad Sci USA 2007; 104:5495-5500.

25 Schmuck EM, Board PG, Whitbread AK, et al. Characterization of the monomethylarsonate reductase and dehydroascorbate reductase activities of Omega class glutathione transferase variants: implications for arsenic metabolism and the age-atonset of Alzheimer's and Parkinson's diseases. Pharmacogenet Genomics 2005; 15:493-501.

26 Hughes TA. Regulation of gene expression by alternative untranslated regions. Trends Genet 2006; 22:119-122.

27 Pesole G, Mignone F, Gissi C, Grillo G, Licciulli F, Liuni S. Structural and functional features of eukaryotic mRNA un- 
translated regions. Gene 2001; 276:73-81.

28 de la Mata M, Alonso CR, Kadener S, et al. A slow RNA polymerase II affects alternative splicing in vivo. Mol Cell 2003; 12:525-532.

29 Kornblihtt AR. Promoter usage and alternative splicing. Curr Opin Cell Biol 2005; 17:262-268.

30 Monsalve M, Wu Z, Adelmant G, Puigserver P, Fan M, Spiegelman BM. Direct coupling of transcription and mRNA processing through the thermogenic coactivator PGC-1. Mol Cell 2000; 6:307-316.

31 Kishi T, Grass L, Soosaipillai A, Shimizu-Okabe C, Diamandis EP. Human kallikrein 8: immunoassay development and identification in tissue extracts and biological fluids. Clin Chem 2003; 49:87-96.

32 Shimizu C, Yoshida S, Shibata M, et al. Characterization of recombinant and brain neuropsin, a plasticity-related serine protease. J Biol Chem 1998; 273:11189-11196.

33 Tomizawa K, He X, Yamanaka H, Shiosaka S, Yoshida S. Injury induces neuropsin mRNA in the central nervous system. Brain Res 1999; 824:308-311.

34 Matsumoto-Miyai K, Kitagawa R, Ninomiya A, Momota Y,
Yoshida S, Shiosaka S. Decidualization induces the expression and activation of an extracellular protease neuropsin in mouse uterus. Biol Reprod 2002; 67:1414-1418.

35 Chen ZL, Momota Y, Kato K, et al. Expression of neuropsin mRNA in the mouse embryo and the pregnant uterus. $J$ Histochem Cytochem 1998; 46:313-320.

36 Oka T, Akisada M, Okabe A, Sakurai K, Shiosaka S, Kato K. Extracellular serine protease neuropsin (KLK8) modulates neurite outgrowth and fasciculation of mouse hippocampal neurons in culture. Neurosci Lett 2002; 321:141-144.

37 Hirata A, Yoshida S, Inoue N, et al. Abnormalities of synapses and neurons in the hippocampus of neuropsin-deficient mice. Mol Cell Neurosci 2001; 17:600-610.

38 Oka T, Hakoshima T, Itakura M, et al. Role of loop structures of neuropsin in the activity of serine protease and regulated secretion. J Biol Chem 2002; 277:14724-14730.

39 Komai S, Matsuyama T, Matsumoto K, et al. Neuropsin regulates an early phase of schaffer-collateral long-term potentiation in the murine hippocampus. Eur J Neurosci 2000; 12:1479-1486. 